\title{
Integrated Rocket Simulation of Internal and External Flow Dynamics in an e-Science Environment
}

\author{
Soon-Heum Ko, Sangho HAN, Jin-ho KIM and Chongam KIM* \\ Department of Aerospace Engineering, Seoul National University, Seoul 151-742 \\ Jong Bae Moon and Kum Won $\mathrm{CHO}^{\dagger}$ \\ Korea Institute of Science and Technology Information, Daejeon 305-333 \\ Yoonhee $\mathrm{KIM}^{\ddagger}$ \\ Department of Computer Science, Sookmyung Women's University, Seoul 140-742
}

(Received 2 Ocotber 2008, in final form 16 March 2009)

\begin{abstract}
The internal and external flowfield variation of a launch vehicle has been simulated in an e-Science environment. To analyze the igniting process of a solid-rocket propellant, a fluid-structure interaction code has been developed using an ALE (arbitrary Lagrangian Eulerian) kinematical description and a staggered fluid-structure interaction algorithm. Also, unsteady motion of a detached rocket booster has been predicted by using an external flow analysis with an aerodynamic-dynamic coupled solver. A Korean e-Science environment designed for aerospace engineering, e-AIRS [15], supplies a user-friendly interface for such individual work and it can advance to an integrated rocket simulation of internal combustion and external flow variation by controlling the execution and data flow of two flow solvers. As a consequence, e-Science facilitates multi-disciplinary collaborative research, and makes individual work more convenient.
\end{abstract}

PACS numbers: $47.85 . \mathrm{Gj}, 45.40 . \mathrm{Gj}, 47.90 .+\mathrm{a}$

Keywords: e-Science, e-AIRS, Launch vehicle, Fluid-structure interaction, Aerodynamic-dynamic coupled analysis

DOI: $10.3938 / \mathrm{jkps} .55 .2166$

\section{INTRODUCTION}

A rocket system is a system-intensive product that eventually requires the integration of the highest technologies in each discipline, such as fluid physics, structures, propulsion systems, control, and so on, as depicted in Figure 1. Thus, a multi-disciplinary integrated simulation based on accurate computations for each discipline is a key element for successful research and development of a rocket system.

However, only segregated works in each discipline have been tried so far, mainly because it is very hard to obtain an accurate analysis for a single work. As an example of a propulsion system, the ignition process of a solid-rocket propellant in the combustion chamber shows complex multi-physical phenomena due to the non-linear viscoelasticity of a propellant grain and the hot emission gas formed during the burning process. Thus, the researcher should be well aware of the characteristics of heat and

*E-mail: chongam@snu.ac.kr; Fax: +82-2-887-2662

†E-mail: ckw@kisti.re.kr

‡E-mail: yulan@sookmyung.ac.kr high-pressure flow physics, the combustion mechanism of a propellant, and the deformation of the propellant grain due to the structural load and combustion. As another example, an external flow analysis examines aerodynamic forces and moments of a rocket system with dynamic variation, often including relative motion of detached bodies such as exhausted rocket boosters. Thus, in-depth knowledge of compressible flow physics, rigid

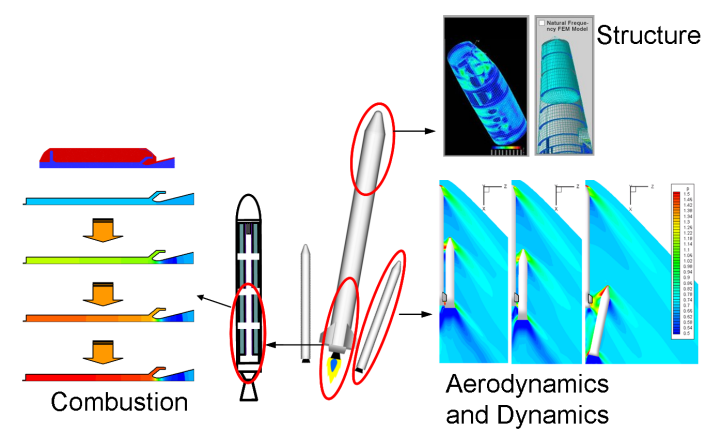

Fig. 1. Individual research for research and development of a launch vehicle. 
body dynamics, and the interference effect of bodies is required.

Considering the complexity of each work, it is far more efficient to build a virtual organization to control collaborative works than to let an individual researcher undertake the whole integrative analysis. To this end, we adopted high-end e-Science technology to construct a cyber workbench for integrated rocket simulations. EScience increasingly represents the global collaborations of people and shared resources to solve new and challenging problems in science and engineering [1] on the basis of an IT infrastructure, typically referred to as the Grid [2]. The infrastructure serves the integrated utilization of computational and experimental facilities, valuable datasets, knowledge, and so on in a secure and transparent manner; thus, scientists/engineers can exploit the e-Science environment for challengeable research [3], such as large-scale computation for complex physical mechanisms [4], combined works of computation and experiments for design-to-development processes [5], and dataintensive research [6].

This paper describes the utilization of an e-Science environment to launch vehicle simulations. As valuable component-wise research, individual studies on the ignition process of a solid-rocket propellant and the separation motion of a strap-on booster due to external flow variation are addressed in Sections 2 and 3. A coupled simulation of internal and external flowfields with the aid of an e-Science environment is then presented in Section 4. Finally, individual and integrated rocket simulations are summarized in Section 5.

\section{INTERNAL FLOW SIMULATION OF A SOLID-ROCKET PROPELLANT}

\section{Motivation}

Solid propellant rockets produce a thrust force by burning contained propellant grain in the combustion chamber and ejecting hot combustion gas through a supersonic nozzle. During the ignition process, complex multi-physical phenomena develop in the interior of the combustion chamber due to the non-linear viscoelasticity of a propellant grain and the hot exhaustion gas formed during the burning process. The main factors that govern the interior phenomena of the solid-rocket are the flow of hot and high-pressure gases, and the deformation of the propellant grain due to the structural load and combustion. Also, each principal element (fluid, structure, combustion) governing complex physical phenomena in the combustion chamber induces a feedback cycle, thus influencing one another.

Because of the physical complexity and strong interaction of multiple disciplines, many previous research efforts have been based on instrumental experiments, and numerical analyses have been conducted just to show the detailed features of a specific local physical domain. However, experimental research is very dangerous and costly to perform, and it is not easy to measure all necessary physical quantities. Therefore, coupled simulation, by integrating the fluid, structure, and combustion parts, is required to address the highly complex unsteady phenomena in a combustion chamber.

\section{rithms}

\section{Governing Equations and Numerical Algo-}

The arbitrary Lagrangian Eulerian (ALE) kinematical description $[7,8]$ has been incorporated into the fluid/solid formulation to accurately capture the deformation of the solid propellant grain. The ALE description is a hybrid approach that combines the advantages of the classical Lagrangian and Eulerian formulations. In the Lagrangian approach, mesh nodes are attached to continuum particles and move with them. In the Eulerian approach, however, nodes of the computational mesh remain fixed, and continuum particles pass through them. Thus, it is clear that neither the Lagrangian nor the Eulerian approach may efficiently describe the progress of burning a solid propellant because some particles are eroded due to the regression of the propellant surface. In the ALE approach, numerical simulations are first conducted in an Eulerian manner, and deformation of the computational mesh is described in the Lagrangian pattern. It is, thus, suitable for simulating the regression of the solid propellant.

Governing equations for fluid simulation are the twodimensional axisymmetric Euler equations in ALE form. Inviscid governing equations can provide an accurate solution as mass flux at exposed grain surface will sweep away the boundary layer. For the structural analysis, the two-dimensional dynamic finite element formulation using the principle of virtual work is transformed into an ALE kinematical description. Also, the mechanical response of the grain is simulated using the ArrudaBoyce nonlinearly elastic constitutive model [9], which has been shown to be quite successful in capturing the small- and large-strain response of filled elastomers. As a combustion modeling, the one-dimensional transient burning model is used to efficiently simulate the burning process at the interface between the fluid domain and the propellant grain surface. It is assumed that the grain is heated by the hot gas in a chamber, and that the ignition process takes place on its exposed surface when the surface temperature exceeds a specific value. Finally, for the adjustment of interchanging information between the fluid and the solid domains, we adopted the loosely coupled conventional sequential staggered scheme suggested by Park \& Felippa [10].

The resulting solver is then validated by conducting a numerical simulation for the problem of the solidpropellant rocket interior and by comparing the com- 


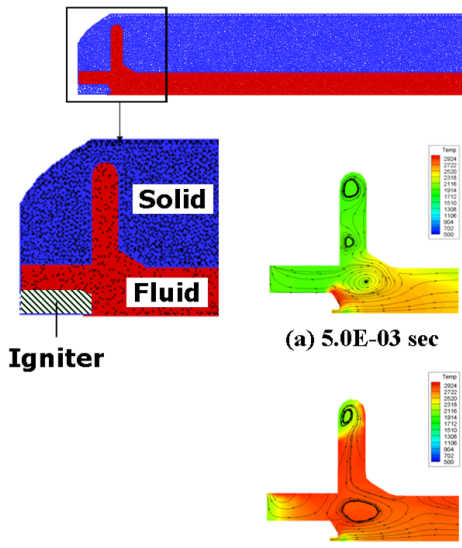

(c) 1.5E-02 sec
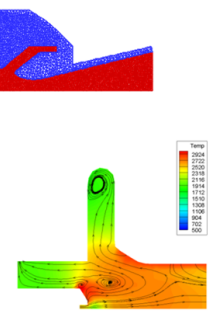

(b) 1.0E-02 sec

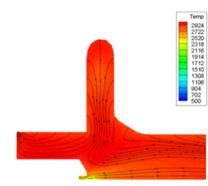

(d) 2.0E-02 sec
Fig. 2. Burning and flow development process as in the forward areas of the combustion chamber.

putational results with the experimental data. Details on the validation process are given in Ref. 11 .

\section{Numerical Results}

The computational domain and the initial burning process near the igniter are shown in Figure 2. A twodimensional unstructured mesh system consisting of a solid propellant and an internal flow region is generated to represent an axisymmetric solid-rocket interior with a pyrogen igniter attached to it. Also, contours at the bottom right show variations of temperature just after the igniter has spouted out the flame. As shown in Figure 2(a), the hot gas ejected from the igniter starts to develop a flow in the combustion chamber. This combustion gas first heats the exposed grain surface near the trimmed slot surface, so the solid grain in this region starts to ignite and send out a mass flux (Figure 2(b)). As the combustion proceeds, the flame propagates very rapidly to the exit direction along the grain surface while this propagation is rather delayed inside the slot where initial cold air is congested (Figure 2(c)). Finally, hot emission gas fills the entire chamber and the whole grain surface produces a mass flux, as in Figure 2(d).

During the combustion process, the propellant grain decreases and is deformed because of the burning mechanism and the structural load from the high-pressure flow in the combustion chamber. Figure 3(a) shows the geometric change of a solid propellant grain 0.786 seconds after the pyrogen igniter ignited. At that time, about 14 $\%$ of the contained propellant was consumed for propulsion. Figure 3(b) shows the pressure history near the forehead of the rocket interior. The pressure in the combustion chamber increases instantly and reaches a peak point. At that time, the nozzle membrane, which is designed to obstruct the outflow until the pressure loading reaches some specific value, is broken, and the confined
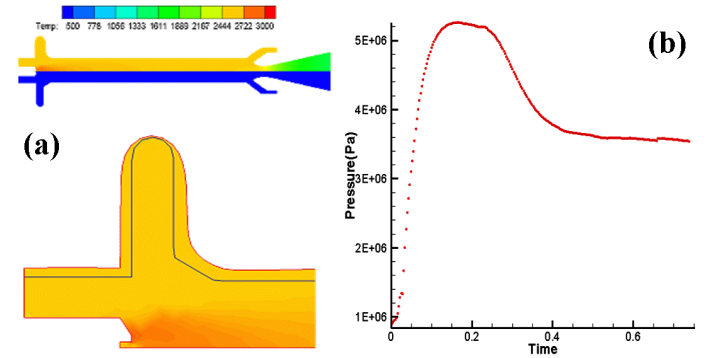

Fig. 3. (a) Temperature contours and change of fluid domain at $t=0.786 \mathrm{sec}$, compared to initial state, (b) Pressure history at $0.15 \mathrm{~m}$ below the exit of an igniter.

flow can spout out through the nozzle. Thus, the pressure in the combustion chamber decreases rapidly until the incoming mass flow from the burned grain and the outgoing mass flow through the nozzle are balanced. After that, the internal gas pressure shows a gradual decrease caused by the volumetric expansion of the fluidic domain due to the consumption of the propellant.

\section{EXTERNAL FLOW VARIATION AROUND A ROCKET SYSTEM}

\section{Motivation}

Strap-on boosters have traditionally been used to increase the payload of launch vehicles. From an aerodynamic point of view, the optimal shape is the one that has minimal drag during flight, and the designed rocket engine is equipped in the booster. However, an optimal booster shape for steady flight does not guarantee a safe separation of the strap-on booster. That is, the detached empty booster after completion of the combustion process may sometimes have a catastrophic collision against the core rocket during free-fall. Hence, it is important to investigate in details of the separation behavior of strapon boosters and to reflect on their aerodynamic-dynamic characteristics during the design process.

Many valuable research projects have been conducted to exploit the aerodynamic-dynamic behavior of launch vehicle configurations during the separation stage $[12$, 13]. However, most previous numerical simulations were insufficient in accurately showing the unsteady flow patterns around the vehicle and the dynamics of detached boosters. Most studies simulated quasi-steady flowfields at predefined positions by using wind tunnel experiment or, when an aerodynamic-dynamic-coupled analysis was conducted, by using inviscid flow simulations conducted without considering the base flow effect. Thus, the current study focuses on accurately predicting the dynamic behavior of detached boosters in an unsteady flowfield by adopting an aerodynamic-dynamic-coupled approach and by conducting a turbulent flow analysis in the broad spatial region around the launch vehicle. 


\section{niques \\ 2. Governing Equations and Numerical Tech-}

Three-dimensional compressible full Navier-Stokes equations are adopted to accurately resolve the massive flow separation in the base region. For an adequate description of the turbulent flowfield within the framework of the RANS (Reynolds-averaged Navier-Stokes) formulation, the k- $\omega$ SST (shear stress transport) two-equation turbulence model is implemented as flow solver. As for numerical techniques, the AUSMPW+ (modified AUSM using pressure-based weight functions) flux scheme with the MUSCL (monotone upstream-centered schemes for conservation laws) approach and the LU-SGS (lowerupper symmetric Gauss-Seidel) method are used for spatial discretization and implicit time integration, and dual time stepping is employed to obtain a second-order accuracy at the time domain.

The relative motion of bodies is predicted by solving six-degree-of-freedom rigid-body equations of motion. In a coupled solver, the result of the aerodynamic force from flow simulation at a former physical time is summed with gravity and returns an acceleration and an angular acceleration by solving Eulers equations of motion. Linear and angular displacements are easily acquired by integrating the former results. Then, the positions of bodies are updated, and fluid analysis is conducted again. All of the details on the above techniques are given in Ref. 14 .

\section{Numerical Results}

The aerodynamic characteristics of the KSR-III (Korean sounding rocket) and the detachment motion of a strap-on during the separation process are examined. KSR-III is a rocket system researched by the Korean Aerospace Research Institute. The application configuration has a core rocket with two strap-on boosters attached to it, as seen in Figure 4 of the overset mesh system, which has about 3.5 million mesh points in total. The freestream Mach number and the Reynolds number are 1.7 and $1.431 \times 10^{7}$, respectively. The flight angle with respect to the ground is 90 degrees, and the angle of attack is zero. The flow properties of the plume gas are obtained by using the chamber condition and cold gas assumption.

Figure 5 shows the trajectory of a separated rocket booster and the pressure field at free separation. The result shows various compressible flow physics that cause a complex dynamic pattern for the detached booster. The first principal factor is the bow shock from the booster nose. After hitting the core rocket, a shock wave turns back to the booster and acts to increase the repulsive force and to generate a positive pitching moment. The next factor is propagated oblique shocks from the fin and flare skirt of the core rocket, which cause a negative pitching moment and an increment in the normal

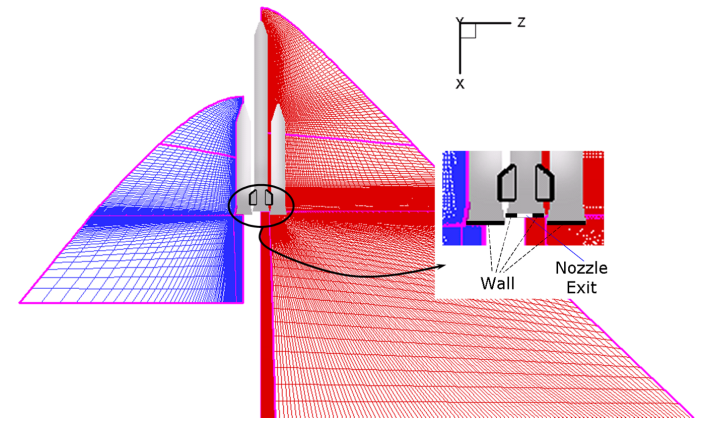

Fig. 4. Overset mesh system around a launch vehicle configuration.

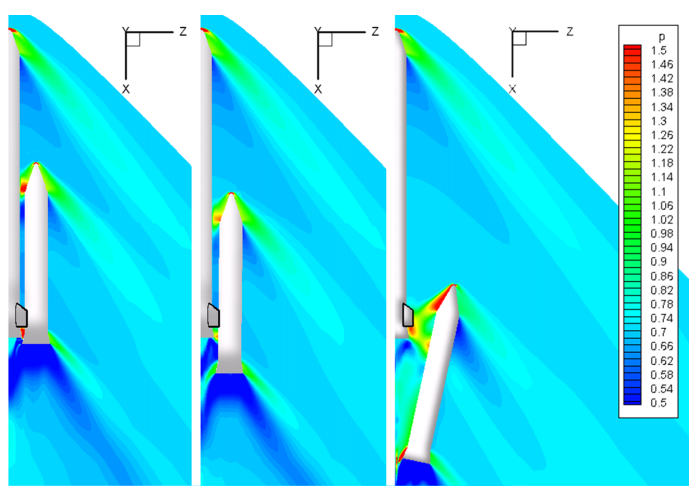

Fig. 5. Separation motion analysis of a launch vehicle (from 0.00 to $0.40 \mathrm{sec}$, with a time interval of $0.20 \mathrm{sec}$ ).

force. Finally, expansion waves from the baseline of the core rocket change the aerodynamic characteristics of the strap-on in the form of a counterbalancing force with the effect of oblique shocks from the core rocket. All things combined, the strap-on initially experiences a negative pitching moment and starts a counterclockwise rotation. However, as the nose of the booster gets closer to the core rocket, the reflected bow shock becomes stronger and generates a strong repulsive force. At the same time, the oblique shock from the core rocket to the bottom of the booster gradually becomes weaker as the traveling distance of the oblique shock increases. These processes make the booster begin a reverse rotation. When the booster eventually gains a positive inclination angle, it tends to accelerate in a positive pitching motion mainly because the asymmetric bow shock of the nose produces a strong positive pitching moment. In addition, the negative pitching moment induced by the oblique shock from the core rocket is sufficiently diminished because the downward motion of the booster reduces the effective length of the moment arm with respect to the boosters center of gravity. Thus, the current configuration finally shows a safe separation.

\section{INTEGRATED ROCKET SIMULATION IN THE E-SCIENCE ENVIRONMENT}




\section{Motivation}

Even though internal and external flow analyses can be successfully conducted using individual solvers, it is still hard to do integrative simulations for the accurate design and development of a rocket system. In a conventional way, a multi-disciplinary analysis by collaboration of researchers includes regular offline meetings, coordination of their ideas, and the sharing and unification of their numerical tools. First, unification of different codes requires such time-consuming and tedious jobs as the unification of variables, I/O formats, etc., and frequent offline meetings. Moreover, opening researchers source codes to other collaborators means that developers face the risk of leaking or abuse of their intellectual property by other researchers or illegal intruders.

This restrictions of the conventional research environment have motivated current researchers to implement an integrative rocket-simulation workbench on e-AIRS (e-Science Aerospace Integrated Research System). EAIRS has been showing its usefulness in fluid dynamic lectures and academic research, on the basis of stabilized infrastructure and convenient user interfaces. To develop a secure integrative rocket-simulation system, current research fully utilizes e-AIRS middleware and minimizes domain scientist's efforts.

\section{Construction of an e-Science Environment} for Rocket Simulation

The procedure of integrated rocket simulation is briefly schematized in Figure 6. As this figure shows, an integrated rocket simulation can be conducted by exploiting the outgoing flow through the nozzle of a combustion chamber as the plume condition for an external flow analysis. In the simulation process, the internal combustion solver first starts until a solid propellant produces a stable thrust. Then, the flow properties at the exit of the nozzle are transferred to an external solver, and an external flow solver concurrently starts by using these properties as plume conditions. In an unsteady simulation, the internal solver advances independently from the external solution procedure, while the external solver gets the plume condition at a given time from an accumulated intermediate dataset of an internal analysis or waits for the result to come.

To construct this research workbench, application codes need only to include the $\mathrm{I} / \mathrm{O}$ routine and tune non-dimensionalization for the exchange of plume conditions. On the other hand, some additional computer scientific modules are to be added to the current e-AIRS system. Basically, this work space uses core technologies implemented on the e-AIRS CFD Service, such as job submission and metadata management systems, schedulers, monitoring tools, etc., that are described in detail in Ref. 15. The one exception is that application solvers

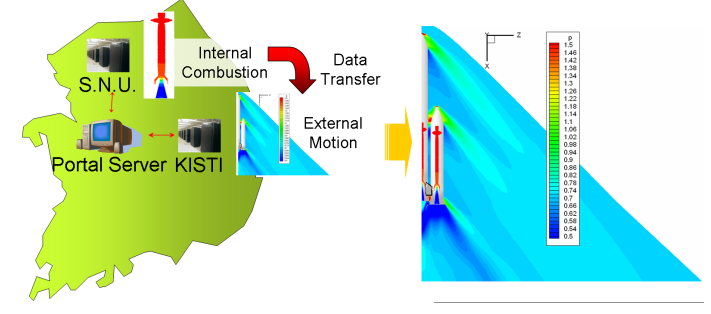

Fig. 6. Procedure of the integrated rocket simulation.

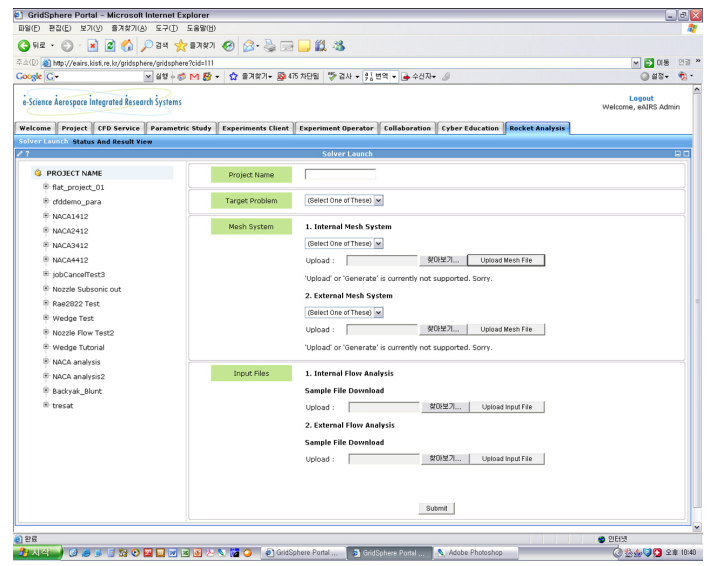

Fig. 7. Portal interface of integrative and individual rocket simulations.

are maintained by code developers, and this makes the current system more complex. As application solvers of internal combustion and external flow are reposited and executed at owners' sites, a middleware module that transmits intermediate data between the two sites is developed using Grid FTP, and another module, which controls the execution process of binary codes, is developed using GT4 technologies. Also, a portal interface for integrated rocket simulation is newly developed.

\section{Utilization}

Figure 7 shows the portal interface of integrated and individual rocket analyses. By choosing a target application, the user can either conduct an individual simulation of internal combustion or external flow analysis, or try an integrated simulation of full flowfields. To apply various flow/numerical conditions, users can manually change an input text file and upload it. As for the mesh system, the current interface only permits use of the default mesh system, due to the generalization problem in the $\mathrm{I} / \mathrm{O}$ format of application codes. When a job is requested, a job execution signal is sent to a fixed site where an application code is stored, and e-Science controls the execution and data transmission of this job.

Figure 8 shows the steady-state pressure contours of integrated simulations for two rocket geometries, are with and are without fins. A quasi-steady solution from 


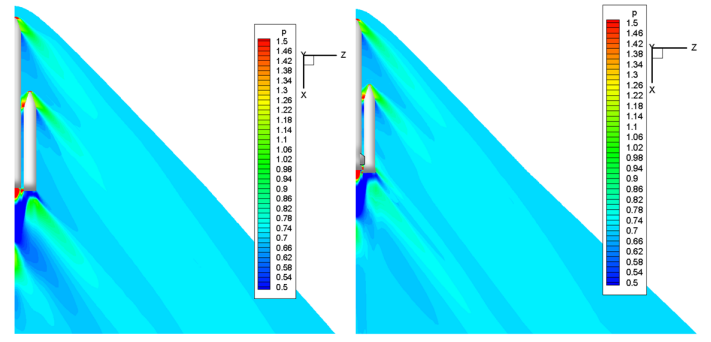

Fig. 8. Steady-state pressure contours of integrated simulations for slender and finned geometries.

the internal analysis is used as the exit condition of the rocket nozzle in the external flow analysis. Overall, the two simulations show a stronger plume emission than was seen in the previous external flow analysis, where the plume was assumed to be a cold gas. As a result, higher pressure is seen at the bottom of a booster, and it increases the negative pitching moment, which brings about a counterclockwise rotation of the strap-on booster. Consequently, the booster is presumed to show a more unsafe motion during separation than is seen with the cold-plume gas simulation.

\section{CONCLUSIONS}

Integrated and individual rocket simulations of internal combustion and external flow variation are conducted in an e-Science environment. An internal combustion solver is used to analyze the ignition process of a solidrocket propellant by integrating fluid and structure analysis tools, and combustion modeling. The result describes the details of the initial burning and flame propagation process of the exposed propellant grain surface and the structural deformation of a solid propellant grain due to the effect of the hot, and high-pressure gas inside the chamber. For an external flow analysis with a separation motion simulation of rocket boosters, the aerodynamic solution procedure is coupled with rigid body dynamics. The result shows complex flow physics around a launch vehicle and the influence of various factors on unsteady motion of strap-on boosters. Finally, a workbench for rocket simulations was developed in the e-Science environment. Additional computer scientific modules were implemented on an existing e-AIRS system, and integrative simulations were successfully conducted in an eScience environment.

\section{ACKNOWLEDGMENTS}

The current work is a product of the Korea National e-Science project. The authors are grateful to the Korea Institute of Science and Technology Information for their financial support. Also, the authors appreciate the financial supports provided by NSL(National Space Lab.) program throgh the National Research Foundation of Korea funded by the Ministry of Education, Science and Technology (Grant 20090091724) and the authors are grateful to the Agency for Defence Development for financial support on solid-rocket propellant research. Additional support from the Institute of Advanced Aerospace Technology at Seoul National University is also appreciated.

\section{REFERENCES}

[1] T. Hey and A. E. Trefethen, Grid Computing - Making the Global Infrastructure a Reality, edited by F. Berman, G. Fox and A. J. G. Hey (Wiley, New York, 2003).

[2] The Grid: Blueprint for a New Computing Infrastructure, edited by I. Foster and C. Kesselman (Morgan Kaufmann, San Francisco, 1999).

[3] K. Cho, J. Korean Phys. Soc. 53, 1187 (2008).

[4] H. Katayama-Yoshida, K. Sato, T. Fukushima, M. Toyoda, H. Kizaki, Van An Dinh and P. H. Dederichs, J. Korean Phys. Soc. 53, 1 (2008).

[5] H. Y. Choi and J. U. Kim, J. Korean Phys. Soc. 53, 1388 (2008).

[6] J. Hwang, K. H. Kim, K. S. Cho, Y. D. Park, D. Y. Lee and L. R. Lyons, J. Korean Phys. Soc. 53, 897 (2008).

[7] C. W. Hirt, A. A. Amsden and J. L. Cook, J. Comput. Phys. 135, 198 (1997).

[8] J. Donea, Computational Methods for Transient Analysis, edited by T. Belytschko and T. J. R. Hughes (Elsevier, Amsterdam, 1983).

[9] C. Hwang, L. Massa, R. Fiedler and P. H. Geubelle, 38th AIAA/ASME/SAE/ASEE JPC Conference and Exhibit (Indianapolis, 2002).

[10] K. C. Park and C. A. Felippa, J. App. Mech. 47703 (1980).

[11] S. Han, H. S. Choi, D. H. Min, C. Kim and C. Hwang, Proceedings of the Fifth National Congress on Fluids Engineering (Jeju, 2008).

[12] L. M. Gea and D. Vicker, Computational Fluid Dynamics 2004: Proceedings of the Third International Conference on Computational Fluid Dynamics (Toronto, 2004).

[13] S. J. Choi, C. Kim, O. H. Rho and J. J. Park, J. Spacecraft and Rockets 39, 439 (2002).

[14] S. H. Ko and C. Kim, J. Spacecraft and Rockets 45, 485 (2008).

[15] J. Kim, S. H. Ko, C. Kim, Y. Kim and K. W. Cho, Proceedings of the 6th International Workshop on Challenges of Large Applications in Distributed Environments (Boston, 2008). 